\title{
Diurnal rhythm in endogenous glucose production is a major contributor to fasting hyperglycaemia in type 2 diabetes. Suprachiasmatic deficit or limit cycle behaviour?
}

Received: 28 November 2005 / Accepted: 18 February 2006 / Published online: 16 May 2006

(C) Springer-Verlag 2006

\begin{abstract}
Aims/hypothesis: An increase in endogenous glucose production (EGP) is a major contributor to fasting morning hyperglycaemia in type 2 diabetes. This increase is dissipated with fasting, later in the day. To understand its origin, EGP, gluconeogenesis and hormones that regulate metabolism were measured over $24 \mathrm{~h}$. We hypothesised that EGP, and therefore glycaemia, would demonstrate a centrally mediated circadian rhythm in type 2 diabetes. Subjects and methods: Seven subjects with type 2 diabetes and six age- and BMI-matched control subjects, fasting after breakfast $(08.00 \mathrm{~h})$, underwent a further $24-\mathrm{h}$ fast, with the infusion of $\left[\mathrm{U}-{ }^{13} \mathrm{C}\right]$ glucose and $\left[3-{ }^{14} \mathrm{C}\right]$ lactate, starting at $14.00 \mathrm{~h}$. The MCR and production of total and gluconeogenic glucose were determined from the tracer concentrations using compartmental analysis. Results: MCR was near constant: $1.73 \pm 0.10$ in control and $1.40 \pm 0.14 \mathrm{ml} \mathrm{kg}^{-1} \mathrm{~min}^{-1}$ in diabetic subjects $(p=0.04)$. EGP in diabetes rose gradually overnight from $8.2 \pm 0.7$ to $11.3 \pm 0.5 \mu \mathrm{mol} \mathrm{kg}^{-1} \mathrm{~min}^{-1}$ at $06.00 \mathrm{~h}(p<0.05)$. Glucose utilisation lagged EGP, rising from $8.5 \pm 0.6$ to $10.5 \pm 0.4 \mu \mathrm{mol} \mathrm{kg}{ }^{-1} \mathrm{~min}^{-1}$ $(p<0.05)$, inducing a fall in glycaemia from a peak of $8.0 \pm 0.5 \mathrm{mmol} / 1$ to $6.3 \pm 0.4 \mathrm{mmol} / 1(p<0.05)$. Cortisol and melatonin showed diurnal variations, whereas insulin, glucagon and leptin did not. Melatonin was most closely related to EGP, but its secretion was attenuated in diabetes $(p<0.05)$. Conclusions/interpretation: In type 2 diabetes, EGP and gluconeogenesis display diurnal rhythms that drive the fasting hyperglycaemia and are absent in healthy control subjects. The rise in EGP may be related to a deficit in
\end{abstract}

\author{
J. Radziuk $(\bowtie) \cdot$ S. Pye \\ Diabetes and Metabolism Research Unit, \\ Ottawa Hospital (Civic Campus), \\ 1053 Carling Avenue, \\ Ottawa, ON, K1Y 4E9, Canada \\ e-mail: jradziuk@ohri.ca \\ Tel.: +1-613-7614112 \\ Fax: +1-613-7615329 \\ J. Radziuk - S. Pye \\ Ottawa Health Research Institute and the University of Ottawa, \\ Ottawa, ON, Canada
}

suprachiasmatic nucleus activity in diabetes, or result from non-linear behaviour plus a transition from a normal steady state to a limit cycle pattern in diabetes, or both.

Keywords Diurnal rhythms · Dynamical diseases · Endogenous glucose production · Fasting plasma glucose · Limit cycles $\cdot$ Metabolic clearance $\cdot$ Non-linear systems · Tracer methods - Type 2 diabetes

Abbreviations EGP: endogenous glucose production MCR: metabolic clearance rate (of glucose) $\cdot R_{\mathrm{d}}$ : utilisation rate (of glucose) $\cdot \mathrm{SCN}$ : suprachiasmatic nucleus

\section{Introduction}

Fasting hyperglycaemia is one of the principle criteria for the diagnosis of type 2 diabetes [1]. Its aetiology has been attributed to the beta cell, the liver and the peripheral tissues (muscle) [2], with the relative contributions a matter of continuing investigation.

Since it is the principal source of glucose under postabsorptive conditions, the liver has been implicated as an important player [2,3], with correlations demonstrated between endogenous glucose production (EGP) and fasting glucose concentrations in a large number of laboratories (for review, see [4]). Some uncertainty arose from a number of observations of similar rates of EGP in type 2 diabetes and healthy control subjects $[5,6]$. The resolution of this issue was based on the following observations: (1) glycaemia in type 2 diabetes is never constant, and so the assumption of a steady state could lead to quantitative inaccuracies [7,9]; and (2) EGP is elevated in the morning by $30-50 \%$ and decreases to near-normal rates by afternoon $[4,7,8]$. This results in a high degree of correlation between elevated glycaemia and EGP early in the morning and its dissipation by mid-afternoon [7, 8]. The time of its determination is therefore critical to the conclusions drawn, as is the methodology used. The metabolic clearance rate (MCR) of glucose, however, remains constant throughout the day and is decreased in 
type 2 diabetes [7]. It therefore accounts for the baseline elevation in glycaemia that persists throughout the day [7,9].

Gluconeogenesis has been implicated in the fasting increase in EGP $[10,11]$. When deuterated water and the relative incorporation of the deuterium into the second and fifth position of glucose is used to estimate fractional gluconeogenesis, both gluconeogenesis and glycogenolysis appear to contribute to the elevated fasting EGP [12-15].

The observations discussed lead to the following questions:

1. How does the EGP increase to its morning level from rates that are near normal the previous evening? Does this occur during fasting?

2. Is gluconeogenesis a primary contributor to this increase?

The aim of this study was therefore to investigate the hypotheses that in type 2 diabetes (1) there is a diurnal rhythm in EGP with a nocturnal increase and daytime fall; (2) the changes in EGP drive those in glycaemia; (3) gluconeogenesis is a major contributor to the changes in EGP; and (4) the cyclicity of these processes occurs independently of exogenous influences such as meals, and, therefore, autonomously.

Patients with type 2 diabetes were compared with nondiabetic control subjects in terms of the rate of glucose production and an index of gluconeogenesis, measured using tracers during a 24-h fast. The relationship of the diurnal cycle in EGP with circadian rhythms was examined.

\section{Subjects and methods}

Subjects Eight patients with type 2 diabetes and eight healthy individuals participated in these studies. Control subjects were matched for age, sex and BMI. All diabetic subjects were treated with diet and exercise $(n=4)$, or with sulfonylureas and/or metformin $(n=4)$. Any oral hypoglycaemic agents were discontinued 3 days prior to the study. Other medications used were not considered to affect glucose metabolism. The subjects were otherwise healthy on physical and laboratory examination. The studies were approved by the Human Ethics Review Board of the Ottawa Hospital and participants gave their informed consent.

Protocol Subjects were prescreened using a polysomnogram, with overnight continuous measurement of four- channel electroencephalogram, one-lead electrocardiogram, finger oximetry, gastrocnemius electromyogram, and rib cage and abdominal motion by electrical impedance bands. Subjects with sleep apnoea or other sleep disorders were not included in the study because of the known effects of these conditions on metabolism. On day 1 , subjects fasted after breakfast $(08.00 \mathrm{~h})$. They were admitted to the clinical investigation area at 12:00 $\mathrm{h}$ (day 1). Unprimed infusions of $\left[\mathrm{U}-{ }^{13} \mathrm{C}\right]$ glucose and $\left[3-{ }^{14} \mathrm{C}\right]$ lactate were initiated at $14.00 \mathrm{~h}$. Sleep was again monitored during the night. The study continued until $14.00 \mathrm{~h}$ on day 2 . The initiation and termination times were adjusted relative to the normal bedtimes of the subjects so that approximately $9 \mathrm{~h}$ of sampling was carried out prior to start of sleep (Table 1). Samples were collected for metabolite, tracer and hormone measurements on a variable schedule related to the anticipated rapidity of changes in the tracer concentrations. At night, samples were collected less frequently, but at least every $40 \mathrm{~min}$.

Materials $\left[3-{ }^{14} \mathrm{C}\right]$ lactate was obtained from Amersham (Arlington Heights, IL, USA). $\left[\mathrm{U}_{-}{ }^{13} \mathrm{C}\right]$ Glucose was obtained from Cortec (Paris, France). Labelled glucose was purified using ion-exchange HPLC (HPX-87P column; Biorad, Hercules, CA, USA). The $\left[{ }^{14} \mathrm{C}\right]$ lactate was purified on an HPX-87H column (Biorad) All products were then tested for sterility and pyrogenicity.

Analytical methods Plasma glucose and lactate were measured using the YSI 2300 (Yellow Springs Instrument Company, Yellow Springs, OH, USA). Plasma NEFA concentrations were determined enzymatically (Wako Chemicals, Richmond, VA, USA). Plasma insulin, glucagon (CV 8\%) and leptin were determined using RIAs (with inter-assay CVs of $4 \%, 8 \%$ and $10 \%$, respectively) from Linco Research (St Charles, MI, USA). Melatonin was also measured by RIA (IBL, Hamburg, Germany). Cortisol was measured using an ELISA (Alpco, Salem, $\mathrm{NH}$, USA).

All samples for tracer measurements were prepared by deproteinisation [16]. The supernatant was passed through ion-exchange resin (Dowex 1-X8 and Dowex 50W; Biorad). An aliquot of the neutral fraction was evaporated to dryness and the residue dissolved in water and scintillation fluid added (Scintisafe; Fisher Scientific, Hampton, NH, USA). The lactate was removed from the resin using formic acid $(0.5 \mathrm{~N})$, which was then evaporated to dryness, dissolved in water and scintillation fluid added. The radioactivity was determined in a liquid scintillation

Table 1 Experimental schedule

\begin{tabular}{|c|c|c|c|c|c|c|c|}
\hline & \multicolumn{3}{|c|}{ Start tracer infusion } & \multicolumn{2}{|l|}{ Bedtime } & \multicolumn{2}{|c|}{ Termination of study } \\
\hline & $-9 \mathrm{~h}$ & & & $0 \mathrm{~h}$ & & & $+15 \mathrm{~h}$ \\
\hline & $\downarrow$ & & & $\downarrow$ & & & $\downarrow$ \\
\hline $\begin{array}{l}\text { Time of tracer } \\
\text { infusion (min) }\end{array}$ & 0 & 240 & 480 & 720 & 960 & 1,200 & 1,440 \\
\hline 24-h clock time & 14.00 & 18.00 & 22.00 & 02.00 & 06.00 & 10.00 & 14.00 \\
\hline
\end{tabular}


counter (Tricarb 2200CA; Perkin Elmer, Wellesley, MA, USA) [16].

The enrichment of plasma $\left[\mathrm{U}_{-}{ }^{13} \mathrm{C}\right]$ glucose was determined by derivatising the glucose in deproteinised plasma with butyl boronic acid (Alfa Aesar, Ward Hill, MA, USA) and acetic anhydride (BDH, Poole, UK) in the presence of pyridine (BDH) [17]. The butaneboronyl derivative was analysed on a GC/MS (Agilent Technologies, Palo Alto, CA, USA) using a Zebron 1701 column (Phenomenex, Torrence, CA, USA) and selective ion monitoring at 297 and $303 \mathrm{~m} / \mathrm{z}$, and a standard curve of known enrichments.

Calculations Glucose kinetics were analysed using nonsteady-state methods and compartmental analysis, as detailed previously $[4,7]$. Concentrations of the infused glucose tracer were fitted to integrated exponential functions [18]. The optimal number of exponentials determined the number of compartments used subsequently ( $\chi^{2}$ test). MCR, volume of distribution and, if necessary, exchange parameters were evaluated from the infused tracer and used in the calculation of EGP and the rate of glucose utilisation from the glucose concentrations. MCRs were also allowed to vary if indicated by a runs test [7].

An index of gluconeogenesis was obtained from the incorporation of ${ }^{14} \mathrm{C}$ label from lactate into glucose [19]. The rate of appearance of $\left[{ }^{14} \mathrm{C}\right]$ glucose was calculated in exactly the same way as that of glucose, i.e. using the MCR determined from the infused glucose label. This rate of $\left[{ }^{14} \mathrm{C}\right]$ glucose appearance represents the transfer of labelled carbon from circulating lactate to glucose. To convert it to a mass rate, it was divided by the interpolated lactate specific activity at each time point, yielding a gluconeogenetic rate in $\mu \mathrm{mol} \mathrm{kg} \mathrm{kg}^{-1} \mathrm{~min}^{-1}$. Since this is neither corrected for dilution of label by carbon from the tricarboxylic acid cycle [20], nor accounts quantitatively for all substrates, it is considered an index of gluconeogenesis.

Comparisons of (hormone, metabolite, flux) time course data between diabetic patients and control subjects were made using ANOVA with time as a repeated measure. The Greenhouse-Geisser correction was used to adjust the $p$ values. Contrast analysis was used to compare potential differences in the time profiles of the concentration or flux data between the two groups. Anthropometric data and extracted parameters were compared using $t$-tests, after testing for the equality of variances. One-tailed tests were used where appropriate. An autoregressive moving average model was used to compute cross-correlations between variables (melatonin, cortisol and EGP) that displayed cyclic behaviour, after prewhitening with a filter based on modelling the predictor variable. A cross-correlation function was determined for different lag times in each experiment. SAS (SAS Institute, Cary, NC, USA) software was used. Results are presented as means \pm SEM, and flux rates are calculated per $\mathrm{kg}$ of body weight.

\section{Results}

Subjects The clinical characteristics of the subjects are summarised in Table 2. All polysomnogram results, both before and during the studies, were within normal limits in the patients. In particular, there was no evidence of abnormal periods of sleep apnoea.

Glycaemia In diabetic subjects, glucose concentrations (Fig. 1) initially decreased from $7.2 \pm 0.3 \mathrm{mmol} / 1$ and plateaued near $6.3 \mathrm{mmol} / 1$ for several hours. By midnight (600 $\mathrm{min})$, concentrations had started to increase, and rose continuously until $07.00 \mathrm{~h}(1,020 \mathrm{~min})$, when they peaked at $8.0 \pm 0.5 \mathrm{mmol} / 1$, gradually decreasing thereafter to $6.3 \pm$ $0.4 \mathrm{mmol} / 1$ by the end of the study. In contrast, control

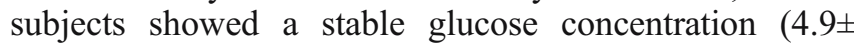
$0.2 \mathrm{mmol} / \mathrm{l}$ ) until near the end of the study, when a drift to about $4.4 \mathrm{mmol} / 1$ occurred. The two glycaemic curves are significantly different $(p=0.0003)$. Contrast analysis of datapoints against a baseline level shows that the profiles of the two curves diverge significantly $(p<0.05)$ at $t=825$ and remain different until $t=1,410 \mathrm{~min}$.

Fluxes The MCR of glucose (Table 3) was near constant and averaged $1.73 \pm 0.10 \mathrm{ml} \mathrm{kg}^{-1} \mathrm{~min}^{-1}$ in control subjects and $1.40 \pm 0.14 \mathrm{ml} \mathrm{kg}^{-1} \mathrm{~min}^{-1}$ in diabetic patients $(p=0.04)$. There was a gradual rise in EGP, from $8.2 \pm 0.7$ to $11.3 \pm$ $0.5 \mu \mathrm{mol} \mathrm{kg}{ }^{-1} \mathrm{~min}^{-1}$ over the course of the night (Fig. 2, Table 3), peaking near $940 \mathrm{~min}(\sim 06.30 \mathrm{~h})$ in diabetic patients. This is in contrast to control subjects, where EGP remained near constant at $8.2 \pm 0.4 \mu \mathrm{mol} \mathrm{kg} \mathrm{min}^{-1}$, before drifting down to $7.2 \pm 0.2 \mu \mathrm{mol} \mathrm{kg}^{-1} \mathrm{~min}^{-1}$, but only after $\sim 24 \mathrm{~h}$ of fasting. The time course of EGP is different in the two groups $(p=0.0017)$, with contrast analysis showing a significant $(p<0.05)$ divergence between $560<$ $t<1,100 \mathrm{~min}$ ( 23.30 and $08.30 \mathrm{~h}$ on day 2 ). The pattern of glucose removal in diabetic subjects $\left(R_{\mathrm{d}}\right.$; Fig. 2$)$ was similar to that for glucose, peaking $\left(10.5 \pm 0.4 \mu \mathrm{mol} \mathrm{kg}{ }^{-1}\right.$ $\left.\mathrm{min}^{-1}\right)$ at $07: 30 \mathrm{~h}$ from a baseline value of $8.5 \pm 0.6 \mu \mathrm{mol}$ $\mathrm{kg}^{-1} \min ^{-1}$. The time course was different in control subjects $(p=0.0017)$, who maintained a removal rate near $8.2 \pm 0.3 \mu \mathrm{mol} \mathrm{kg}{ }^{-1} \mathrm{~min}^{-1}$ for most of the study. The two

Table 2 Subject characteristics

\begin{tabular}{llll}
\hline & $\begin{array}{l}\text { Control } \\
\text { subjects }\end{array}$ & $\begin{array}{l}\text { Type 2 diabetic } \\
\text { patients }\end{array}$ & $p$ value \\
\hline$n$ & 8 & 8 & \\
Age (years) & $49.0 \pm 3.5$ & $53.4 \pm 3.8$ & 0.4 \\
Sex (male/female) & $4 / 4$ & $5 / 3$ & \\
Weight (kg) & $79.7 \pm 4.0$ & $88.6 \pm 5.4$ & 0.2 \\
BMI & $28.8 \pm 1.2$ & $29.7 \pm 1.5$ & 0.6 \\
HbA 1 c & $0.052 \pm 0.001$ & $0.071 \pm 0.006$ & $<0.002$ \\
Fasting plasma & $5.4 \pm 0.2$ & $8.0 \pm 0.6$ & $<0.002$ \\
glucose (mmol/l) & & & \\
Time since & $\mathrm{NA}$ & $5.3 \pm 1.7(0.5-15)$ & \\
diagnosis (years) & & & \\
\hline
\end{tabular}




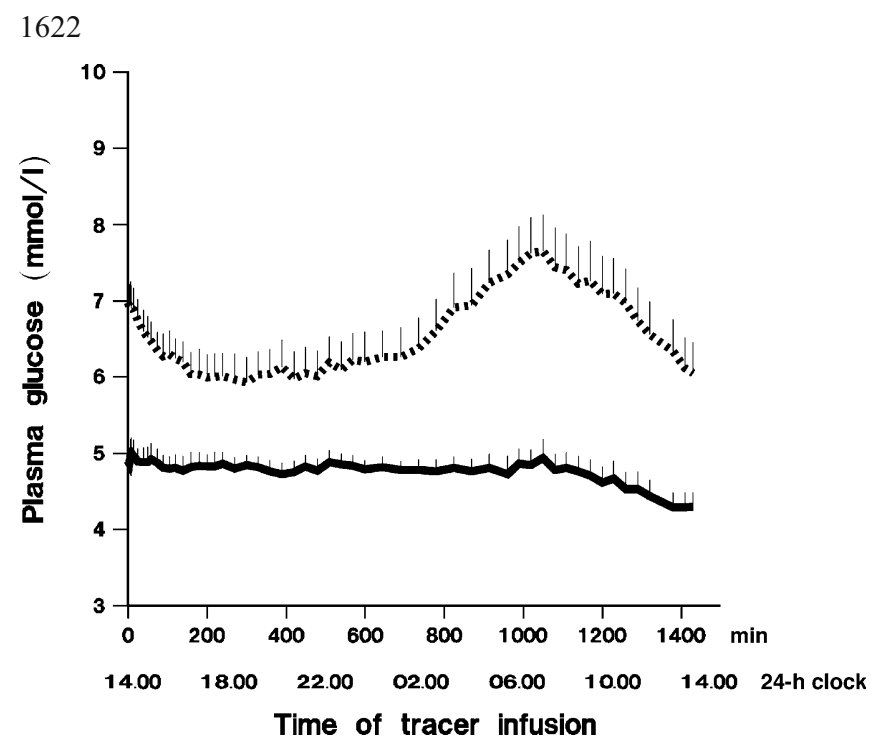

Fig. 1 Glucose concentrations in fasted subjects with type 2 diabetes mellitus (dashed line) compared with control subjects (solid line). The abscissa is given both as the tracer infusion time and the corresponding nominal time on a 24 -h clock

curves diverged significantly $(p<0.05)$ after $t=600 \mathrm{~min}$ (midnight) until the end of the study. The mismatch between EGP and $R_{\mathrm{d}}$ explains the diurnal rise and fall of glucose concentrations in type 2 diabetes.

An index of gluconeogenesis (Fig. 3) was obtained from the rate of incorporation of labelled carbon from $\left[{ }^{14} \mathrm{C}\right]$ lactate into glucose, which was infused simultaneously in these studies. It increased significantly with time in both groups $(p<0.0001)$, representing the well-known rise with fasting. This increase was about $45 \%$ from the beginning to the end of the study ( $24 \mathrm{~h}$ of fasting). Contrast analysis further demonstrated differences in profile between the two groups that were significant at the $p<0.05$ level from 840 $1,020 \mathrm{~min}$. This difference translates into a peak in gluconeogenesis when the control gluconeogenic time course was subtracted from that in diabetic subjects (Fig. 3). This peak, superimposed on a rising baseline, parallels the EGP during its nocturnal rise and morning fall, and strongly suggests that gluconeogenesis is a major contributor to the nocturnal increase in EGP. Concentrations of lactate (Fig. 3), the major glucogenic substrate, were elevated in diabetes $(p=0.0023)$, but neither of the two groups showed an interaction with the time course $(p=0.2)$ that might reflect the additional nocturnal rise in EGP. The concentrations of NEFA (Fig. 3), a promoter of gluconeogenesis, were not different between the two groups $(p=0.4)$.

Hormones Figure $4 \mathrm{a}-\mathrm{c}$ shows glucagon and insulin concentrations and the ratio of the two. Glucagon levels remained near constant throughout the study in both groups $(p=0.06)$, but were elevated in diabetes $(p=0.04)$. The differences in insulin concentrations between diabetic and control subjects did not reach significance, but the levels fell over time in both groups $(p=0.0007)$. This fall was translated into a rising glucagon:insulin ratio $(p=0.006)$, which was otherwise not distinguishable between the two groups $(p=0.8)$. Leptin concentrations showed variation among individual subjects, but a similar

Table 3 Details of flux calculations

\begin{tabular}{|c|c|c|c|c|c|c|c|c|}
\hline \multirow[t]{2}{*}{ Experiment number } & \multirow{2}{*}{$\begin{array}{l}\text { Volume of } \\
\text { distribution (1) }\end{array}$} & \multirow{2}{*}{$\begin{array}{l}\text { Mean MCR } \\
\left(\mathrm{ml} \mathrm{kg}^{-1} \min ^{-1}\right)\end{array}$} & \multicolumn{6}{|c|}{ EGP $\left(\mu \mathrm{mol} \mathrm{kg}{ }^{-1} \min ^{-1}\right)$} \\
\hline & & & $240 \mathrm{~min}$ & $480 \mathrm{~min}$ & $720 \mathrm{~min}$ & $960 \mathrm{~min}$ & $1,200 \mathrm{~min}$ & $1,440 \mathrm{~min}$ \\
\hline \multicolumn{9}{|l|}{ Control subjects } \\
\hline 1 & 17.7 & 1.86 & 8.35 & 8.26 & 8.54 & 8.67 & 7.63 & 7.14 \\
\hline 2 & 21.3 & 1.46 & 8.12 & 7.90 & 7.88 & 8.50 & 7.65 & 6.98 \\
\hline 3 & 27.9 & 2.05 & 9.19 & 9.39 & 8.58 & 8.86 & 8.03 & 7.61 \\
\hline 4 & 31.8 & 1.71 & 8.79 & 9.32 & 8.66 & 9.22 & 7.67 & 7.60 \\
\hline 5 & 26.4 & 1.59 & 7.52 & 7.57 & 7.47 & 7.48 & 6.77 & 6.69 \\
\hline 6 & 19.9 & 1.88 & 8.68 & 8.94 & 8.66 & 8.86 & 8.11 & 7.79 \\
\hline 7 & 14.7 & 2.06 & 9.47 & 9.57 & 8.91 & 7.96 & 7.28 & 7.06 \\
\hline 8 & 30.7 & 1.25 & 5.94 & 6.18 & 6.71 & 6.77 & 6.37 & 5.97 \\
\hline Mean & 23.8 & 1.73 & 8.26 & 8.39 & 8.18 & 8.29 & 7.44 & 7.10 \\
\hline SEM & 2.2 & 0.10 & 0.40 & 0.41 & 0.27 & 0.29 & 0.21 & 0.21 \\
\hline \multicolumn{9}{|c|}{ Type 2 diabetic subjects } \\
\hline 1 & 37.5 & 1.49 & 7.28 & 7.92 & 9.24 & 9.08 & 5.45 & 6.84 \\
\hline 2 & 30.9 & 1.33 & 8.94 & 10.18 & 11.08 & 11.95 & 8.31 & 8.31 \\
\hline 3 & 27.0 & 1.10 & 9.10 & 10.00 & 10.01 & 10.96 & 8.72 & 8.22 \\
\hline 4 & 25.1 & 2.23 & 8.71 & 8.50 & 11.48 & 12.43 & 9.76 & 7.41 \\
\hline 5 & 38.3 & 1.46 & 9.43 & 8.87 & 10.23 & 10.65 & 9.36 & 8.65 \\
\hline 6 & 24.6 & 0.86 & 5.86 & 6.42 & 8.32 & 9.65 & 6.91 & 6.46 \\
\hline 7 & 20.6 & 1.61 & 11.57 & 11.70 & 11.79 & 12.25 & 10.11 & 9.90 \\
\hline 8 & 39.3 & 1.17 & 6.52 & 8.44 & 9.24 & 12.92 & 9.26 & 8.01 \\
\hline Mean & 30.4 & 1.41 & 8.43 & 9.00 & 10.17 & 11.24 & 8.49 & 7.97 \\
\hline SEM & 2.5 & 0.15 & 0.64 & 0.57 & 0.43 & 0.49 & 0.56 & 0.38 \\
\hline
\end{tabular}



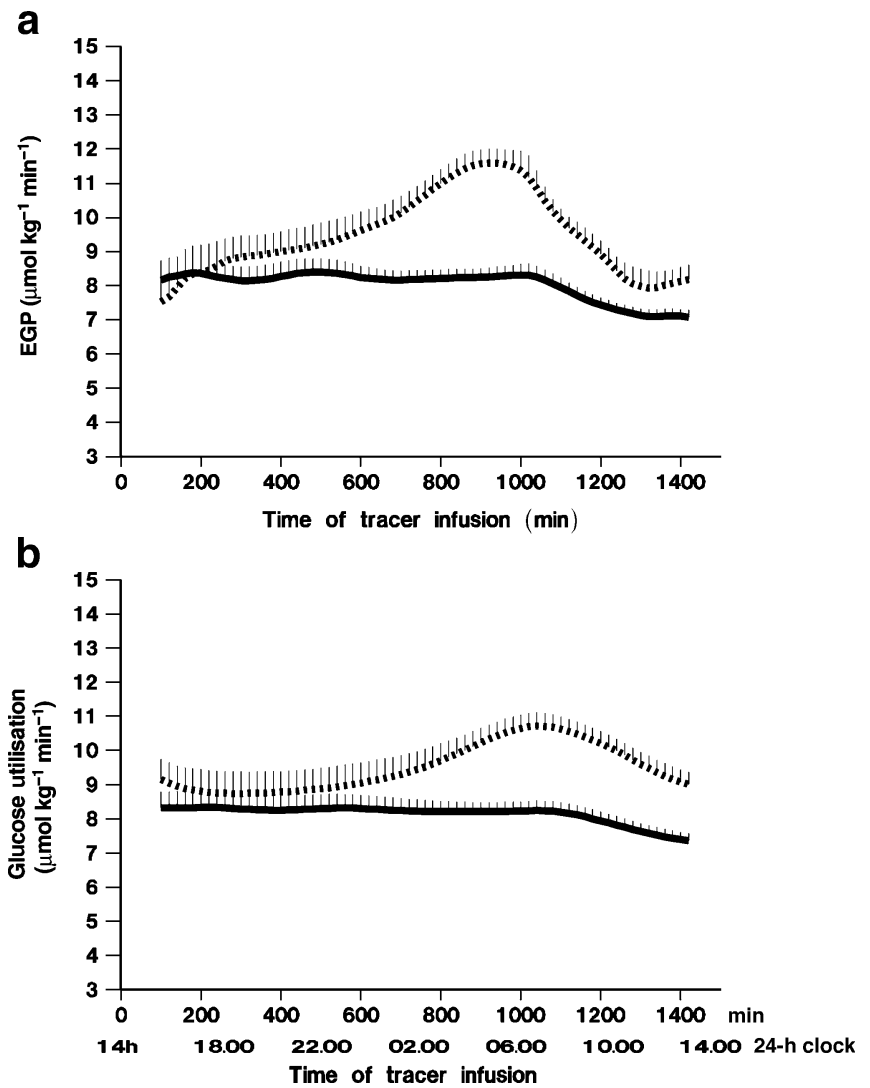

Fig. 2 EGP (a) and glucose utilisation (b) rates in subjects with type 2 diabetes (dashed line) and control subjects (solid line), calculated over the time course of the study using non-steady-state compartmental analysis

overall decrease was seen in the two groups $(p<0.0001)$ during the 24-h study period. The rate of decrease tended to be faster in control subjects (first-order polynomial contrast $p=0.08$ ). Cortisol and melatonin both displayed a cyclicity (changes with time $p=0.006$ for cortisol and $p=0.02$ for melatonin) similar to that in EGP. The time course for cortisol levels was indistinguishable between the diabetic and control groups $(p=0.1$ and contrast analysis). However, the rise of EGP in diabetic subjects precedes the increase in cortisol by $200 \pm 60 \mathrm{~min}$, and the fall in EGP is not accompanied by a corresponding decrease in cortisol. This is consistent with an average cross-correlation function for which the absolute value is less than 0.15 across the spectrum of lag times. Although there is individual variation, the increase in melatonin appears attenuated and delayed in diabetes (vs control subjects). Differences between the two groups are seen for an eighth order polynomial contrast when all the data are used $(p=0.001)$, and for a cubic when only data up to $t=800$ are used $(p=0.01)$. This is consistent with the nearconstant and similar time course of the two curves at the beginning and end of the study, and the night-time excursion where differences between them occur. Crosscorrelation analysis between melatonin levels and EGP demonstrated an average of 0.4 at zero lag time and less than 0.1 for all other lag times, suggesting a degree of synchrony between the two curves.

\section{Discussion}

This study demonstrates a spontaneous diurnal variation of glucose concentrations in type 2 diabetes, with a gradual transition from a nadir in the late evening to a peak in the morning. Such phasic behaviour was not detectable in healthy control subjects. It is, moreover, driven by parallel changes in EGP, specifically, gluconeogenesis. The results answer the question of the provenance of the morning increase in EGP, and a significant portion of the hyperglycaemia. Juxtaposition with studies demonstrating a repeated cycle in EGP during a near isoglycaemic clamp in diabetes [21] over a 3-day period, strongly suggest that the 24-h pattern of EGP can be extended to conditions where the EGP provides the hyperglycaemic drive. Since subjects were fasting from the previous morning, it is highly unlikely that the morning fasting hyperglycaemia depends on preceding meals. The lack of a detectable increase in insulin secretion in response to the nocturnal rise in glycaemia further accentuates this rise, and emphasises the glucose insensitivity of insulin secretion (which might also undergo diurnal exacerbations) in the diabetic state.

These results can be explained by:

1. A circadian rhythm in EGP, probably driven by the suprachiasmatic-paraventricular axis and mediated by the autonomic nervous system and perhaps by hormones such as cortisol; and

2. Limit cycle behaviour: a conversion from normal to diabetes, characterised by a transition from a stable steady state to another stable but cyclic state (of EGP and glycaemia), in a metabolic system that is inherently complex and non-linear.

Circadian rhythms in glucose metabolism and the suprachiasmatic nucleus Studies on variations in glucose levels have produced a range of results. One study reported diurnal variations in the concentrations and fluxes of glucose and other metabolites in diabetic, but not control, subjects [22]; another found elevated morning glucose levels in both diabetic and control subjects, although these were significantly attenuated in the latter group [23]; and still others observed no additional increase in glycaemia in overnight-fasted diabetic subjects [24, 25]. These nocturnal increases in glucose may be related to the dawn phenomenon [26, 27], although this usually starts later than the rise in EGP described here and is likely related to postprandial counter-regulatory effects. The precise glycaemic profile seen could therefore depend on the preceding meal schedule and content [28]. The observations in the present study, however, are independent of meals because of the extended fasting period. Daily variations in metabolism have been related to the hypothalamic biological clock $[29,30]$. In rodents, a rise 

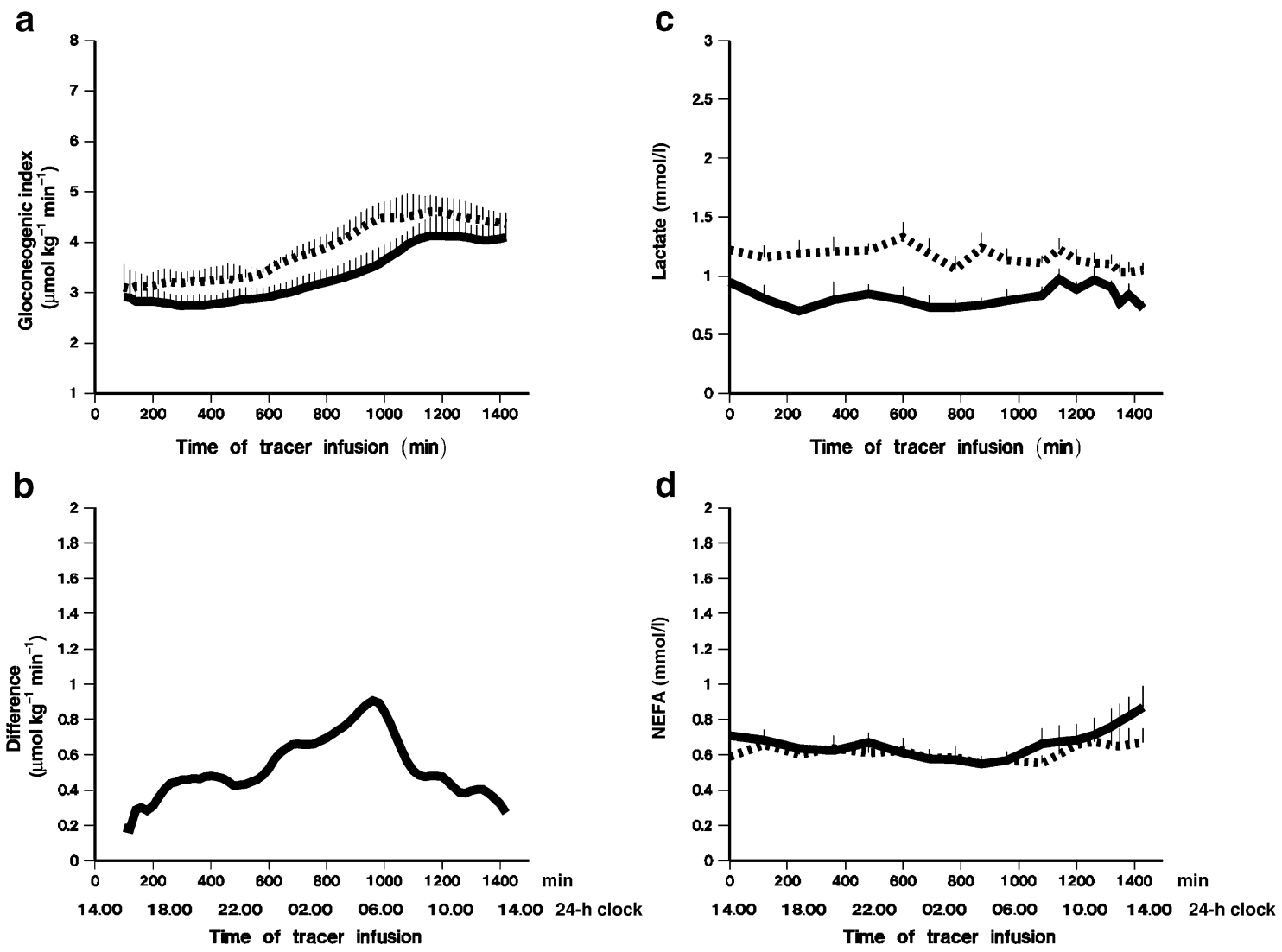

Fig. 3 a Gluconeogenic index calculated from the incorporation of ${ }^{14} \mathrm{C}$ from $\left[{ }^{14} \mathrm{C}\right]$ lactate into glucose. Both control (solid line) and diabetic (dashed line) groups demonstrate a gradual increase in the gluconeogenic index as fasting progresses although this occurs more rapidly in diabetes. b Subtraction of the control index from that

obtained in diabetes reveals a superimposed excursion in the gluconeogenic index in diabetes that matches that in EGP. Lactate concentrations (c) and free fatty acid levels (d) over the 24-h period in control (solid line) and diabetic (dashed line) subjects

in plasma glucose concentrations at dusk (the end of the sleep period) has been shown to result from a rise in EGP $[31,32]$. Elegant studies in mice demonstrated, that this rise in EGP was generated via the suprachiasmaticparaventricular pathway inducing a sympathetic stimulation of EGP [33]. This neural pathway resembles that controlling melatonin secretion: photic information is conveyed from the retina to the suprachiasmatic nucleus (SCN), relayed through the paraventricular nucleus and a multisynaptic pathway to the pineal gland, which secretes melatonin [34], providing the rationale for using melatonin as a surrogate measure for SCN activity in our studies. Although causality cannot be established by analysis of correlations, changes in melatonin concentrations in the present studies are correlated with the changes in EGP in diabetic subjects, suggesting the possibility that this flux, too, may be regulated by the biological clock resident in the SCN. Interestingly, however, the nocturnal excursion of melatonin in the diabetic subjects is postponed and attenuated relative to that in control subjects (Fig. 4), raising the possibility that, unlike the case in rodents, the SCN may be involved in suppressing a cyclic nocturnal increase in EGP, which is then unmasked in diabetes. Parenthetically, the decrease of melatonin itself in diabetes may contribute to the metabolic syndrome, since its ad-

ministration in rats decreased body weight and suppressed visceral fat and plasma leptin [35].

Since SCN activity appears diminished in diabetes, a straightforward linkage of the cycle in EGP (and glycaemia) to the hypothalamic biological clock appears less likely. Furthermore, such an association in rodents relates to arousal and occurs at dusk (dawn phenomenon in humans), with a shorter lived cycle than that seen in our study. It occurs in normal animals, rather than being restricted to diabetes as seen here. The clock-related rise in EGP is also caused by glycogenolysis [36], which is controlled by the sympathetic innervation to the liver [33, 37], whereas gluconeogenesis plays an important part in the increased glucose flux in our study. Although a deficit in SCN activity in type 2 diabetes may explain the changes seen, it is also possible that the circadian association of EGP may be, to some extent, fortuitous.

The rodent model described above [33] also demonstrated peaks in glucocorticoid levels, glucagon or adrenaline, which, although parallel to the glucose excursions, were not responsible for the hyperglycaemia [33]. In our study, only cortisol followed a diurnal pattern (Fig. 4). Its nocturnal rise has been implicated in glucoregulation [38]. Our results show a phase delay with cortisol following increases in both melatonin and EGP (Figs. 2, 


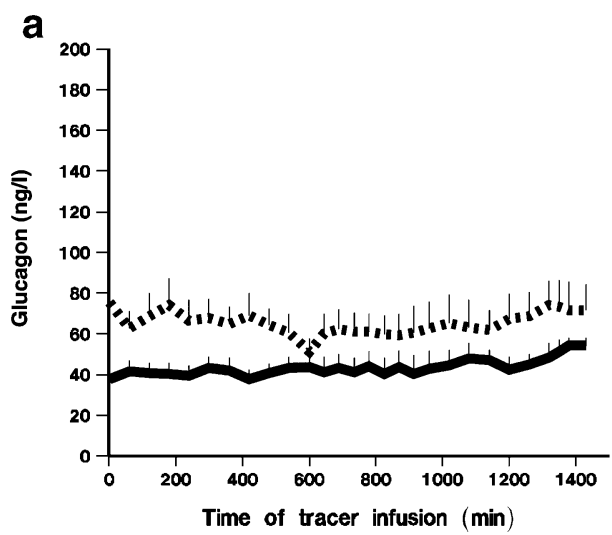

d
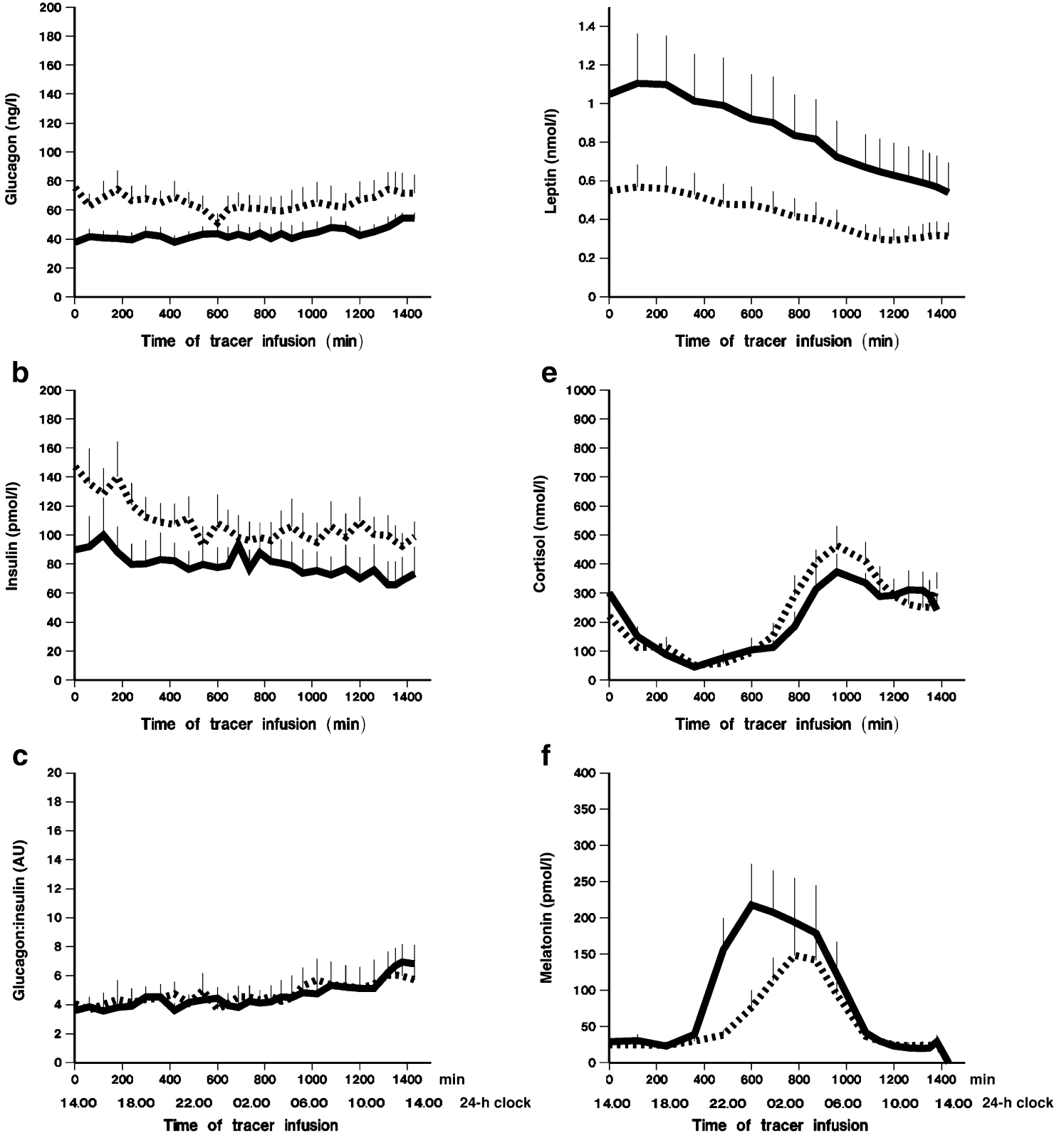

Fig. 4 Hormone levels over the course of the 24-h fast in patients with type 2 diabetes (dashed line) and control subjects (solid line). The panels show the levels of glucagon (a) and insulin (b), the glucagon:insulin ratio (c), and the leptin (d), cortisol (e) and melatonin (f) concentrations

4), obviating a possible role for cortisol in the stimulation of the nocturnal rise in glucose production in diabetes. This is consistent with the lack of correlation of cortisol with EGP also seen here, and with the observation that suppression of corticosterone synthesis did not alter morning hyperglycaemia in diabetes [39].

Limit cycle behaviour: is type 2 diabetes a dynamical disease? It is possible that, in diabetes, insulin resistance (for example) might unmask a more fundamental regulation arising from the SCN. However it remains puzzling why the SCN does not appear to influence glucose fluxes in normal subjects. The need for an alternative explanation is therefore suggested. Variations in EGP have also been seen over a 72$\mathrm{h}$ period in the context of a glycaemic clamp to $11 \mathrm{mmol} / \mathrm{l}$ (near isoglycaemic) in type 2 diabetes [21]. The present study demonstrates that the diurnal changes in EGP drive the corresponding changes in glucose concentrations under physiological fasting conditions. The combination of the two results suggests the stable nature of the cyclic changes in glucose and a degree of autonomy in this rhythm, which would be characteristic of a system property, rather than a response to a regulatory drive.

The existence of two stable states for EGP (and glycaemia), one of which represents a disease state (type 2 diabetes), is highly reminiscent of the description of a 
'dynamical' disease. The concept arises from the generally complex, non-linear nature of many biological systems - a description applicable to the metabolic system. Under appropriate circumstances, the behaviour of such systems can be characterised by the coexistence of dual (or even multiple) stable states. For example, either a steady state (e.g. near-constant glycaemia) or a limit cycle (e.g. a diurnal variation in glycaemia) may occur [40]. When a key control parameter (e.g. an enzyme activity) achieves a critical value, or an appropriate perturbation takes place, the system may pass through a 'bifurcation' or 'switching' point and alter its stable state. Such transitions between stable states are characteristic of dynamical diseases [41, 42] such as Cheyne-Stokes respiration [43] or aplastic anaemia [44]. Interestingly, the onset of type 1 diabetes has been described as a collective dynamic instability within a complex framework [45], rather than a single aetiological factor.

This also appears to be what happens in type 2 diabetes: patients with a (genetic) predisposition to diabetes (and therefore closer to a switching point) may pass from a steady state (constant and normal fasting EGP and glycaemia) to a limit cycle solution (with cyclic changes in EGP and glycaemia and thus elevated morning fasting glucose concentrations) in response to an environmental or lifestyle challenge.

1. Such a cyclic state, which is then necessarily restricted to diabetes, is demonstrated in our study, while explaining an important part of fasting hyperglycaemia.

2. It occurs at the level of gluconeogenesis, which is integrated into the intermediary metabolic network and thus likely to exhibit complex behaviour.

3. Since the transition to the cyclic state represents a 'switch' between two system behaviours, it would be expected to occur rapidly rather than gradually. Indeed, it has been shown that fasting glycaemia does increase abruptly (within a 3-year timeframe) at the onset of diabetes [46]. These complementary data strengthen the argument for non-linear system behaviour. In turn, the transition to a limit cycle provides an explanation for the rapidity of onset of fasting hyperglycaemia.

4. Although it can be influenced by hormones and the central nervous system, the cyclic state is dependent on the entire system and therefore behaves autonomously, explaining both the refractoriness to mealrelated regulation [47] and the inexorable clinical progression of diabetes, which is only delayed by treatment (e.g. [48]).

Overall contribution of fluxes to glycaemia In diabetes, glucose concentrations do not fall to normal levels after the nocturnal excursion has dissipated (Fig. 1). In this group of subjects, the increase over normal at the glycaemic nadir was $1.5 \mathrm{mmol} / \mathrm{l}$ and the increase over this baseline at peak morning values was $1.7 \mathrm{mmol} / \mathrm{l}$. Since EGP is normal at the nadir (Fig. 2), this indicates that at peak values, the decrease in metabolic clearance and the rise in glucose production respectively account for $47 \%$ and $53 \%$ of the increase in glycaemia. This is consistent with previous findings (e.g. [7, 49]). Interestingly, when glucose concentrations were at their lowest in the diabetic subjects, both EGP and $R_{\mathrm{d}}$ were matched and normal (the same as control subjects; Fig. 2), and consistent with a normal glucagon:insulin ratio. The only defect at this point is the low MCR, which necessitates an increase in glycaemia to maintain $R_{\mathrm{d}}$. The increase in EGP, occurring near midnight, then initiates the cycle of increased glycaemia and $R_{\mathrm{d}}$, which allows the return to nadir conditions once EGP decreases.

The gluconeogenic index demonstrates a dual time course. The first concerns the expected increase of about $50 \%$ with fasting, which occurs in both control and diabetic subjects. The value at $22 \mathrm{~h}$ of fasting in control subjects was approximately $3.8 \mu \mathrm{mol} \mathrm{kg}^{-1} \mathrm{~min}^{-1}$. If a correction factor (for label dilution in the tricarboxylic acid cycle) of $\sim 1.5$ is used [20], gluconeogenesis is $70 \%$ of EGP $\left(8.1 \mu \mathrm{mol} \mathrm{kg}{ }^{-1} \min ^{-1}\right)$, almost exactly the same number as found using the deuterated water method in normal subjects [14]. This increase is compensated by a decrease in glycogenolysis (autoregulation) [50]. The second time course involves a cyclic increase in diabetes that corresponds to the rise in EGP, discerned from the difference in the gluconeogenic index between control and diabetic subjects. This is not compensated for by a decrease in glycogenolysis, suggesting a different aetiology for the two gluconeogenic fluxes.

\section{Conclusion}

In conclusion, we have found that EGP in type 2 diabetes is never in steady state, displaying rather a cyclic (diurnal) temporal pattern. This phasic behaviour is restricted to diabetes and is not seen in normal control subjects. It is most closely correlated with melatonin levels and therefore SCN activity, although these are decreased in diabetes, suggesting the possibility of reduced suppression of EGP, a process that might normally be mediated by the SCN. The cyclic behaviour of EGP determines the periodic pattern of glycaemia in diabetes and is, at least partly, determined by the same pattern in the rate of gluconeogenesis, suggesting that this behaviour is fundamentally related to intermediary metabolism. These changes could therefore reflect the intrinsic non-linear behaviour of (primarily hepatic) metabolism that determines glucose levels, but which could also subsume central regulatory pathways. The transition from the normal to the diabetic state would then be characterised by the switch in EGP from steady-state behaviour to the stable cyclic behaviour, demonstrated here.

Acknowledgements The authors gratefully acknowledge the assistance of J. Davies, M. Walczak and A. Baker with these studies, as well as financial support from the Canadian Institutes of Health Research and the Canadian Diabetes Association. We also wish to thank J. Leech for her supervision of the polysomnograms. 


\section{References}

1. American Diabetes Association (2003) Clinical practice recommendations 2003. Diabetes Care 26(Suppl 1):S1-S156

2. DeFronzoR, Bonadonna R, Ferrannini (1992) Pathogenesis of NIDDM: a balanced overview. Diabetes Care 15:318-368

3. Consoli A (1992) Role of liver in pathophysiology of NIDDM. Diabetes Care 15:430-441

4. Radziuk J, Pye S (2002) Quantitation of basal endogenous glucose production in normal and diabetic subjects. Importance of the volume of distribution. Diabetologia 45:1053-1084

5. Hother-Nielsen O, Beck-Nielsen H (1991) Insulin resistance but normal basal rates of glucose production in patients with newly diagnosed mild diabetes mellitus. Acta Endocrinol (Copenh) 124:637-645

6. Jeng C-Y, Sheu WH-H, Fuh MM-T, Chen IY-D, Reaven GM (1994) Relationship between hepatic glucose production and fasting plasma glucose concentration in patients with NIDDM. Diabetes 43:1440-1444

7. Radziuk J, Pye S (2001) Production and metabolic clearance of glucose under basal conditions in type 2 diabetes. Diabetologia 44:983-991

8. Chen Y-DI, Swislocki ALM, Jeng C-Y, Juang J-H, Reaven GM (1988) Effect of time on measurement of hepatic glucose production. J Clin Endocrinol Metab 67:1084-1088

9. Radziuk J, Pye S (2003) Tracer-determined glucose fluxes in health and type 2 diabetes: basal conditions. Best Pract Res Clin Endocrinol Metab 17:323-342

10. Consoli A, Nurijhan N, Capani F, Gerich J (1989) Predominant role of gluconeogenesis in increased hepatic glucose production in NIDDM. Diabetes 38:550-557

11. Magnusson I, Rothman DL, Katz LD, Shulman RG, Shulman GI (1992) Increased rate of gluconeogenesis in type II diabetes mellitus. A ${ }^{13} \mathrm{C}$ nuclear magnetic resonance study. J Clin Invest 90:1323-1327

12. Boden G, Chen X, Stein TP (2001) Gluconeogenesis in moderately and severely hyperglycaemic patients with type 2 diabetes mellitus. Am J Physiol 280:E23-E30

13. Gastaldelli A, Baldi S, Pettiti M et al (2000) Influence of obesity and type 2 diabetes on gluconeogenesis and glucose output in humans. A quantitative study. Diabetes 49:1367-1373

14. Wajngot A, Chandramouli V, Schumann et al (2001) Quantitative contributions of gluconeogenesis to glucose production during fasting in type 2 diabetes mellitus. Metabolism 50:47-52

15. Basu R, Chandramouli V, Dicke B, Landau B, Rizza R (2005) Obesity and type 2 diabetes impair insulin-induced suppression of glycogenolysis as well as gluconeogenesis. Diabetes $54: 1942-1948$

16. Radziuk J, McDonald TJ, Rubenstein D, Dupre J (1978) Initial splanchnic extraction of ingested glucose in normal man. Metabolism 27:657-669

17. Wiecko J, Sherman WR (1976) Boroacetylation of carbohydrates. Correlation between structure and mass spectral behaviour in monoacetylhexose cyclic boronic esters. J Am Chem Soc 98:7631-7637

18. Marquardt DW (1963) An algorithm for least-squares estimation of nonlinear parameters. SIAM J Appl Math 11:431-441

19. Radziuk J (1989) Hepatic glycogen in humans. II Gluconeogenetic formation after oral and intravenous glucose. Am J Physiol 257:E158-E169

20. Hetenyi G Jr (1982) Correction for the metabolic exchange of ${ }^{14} \mathrm{C}$ for ${ }^{12} \mathrm{C}$ atoms in the pathway of gluconeogenesis in vivo. Fed Proc 41:104-109

21. Boden G, Chen X, Urbain JL (1996) Evidence for a circadian rhythm of insulin sensitivity in patients with NIDDM caused by cyclic changes in hepatic glucose production. Diabetes 45:1044-1050
22. Faiman C, Moorhouse JA (1967) Diurnal variation in the levels of glucose and related substances in healthy diabetic subjects during starvation. Clin Sci 32:111-126

23. Shapiro ET, Polonsky KS, Copinschi G et al (1991) Nocturnal elevation of glucose levels during fasting in noninsulindependent diabetes. J Clin Endocrinol Metab 72:444-454

24. Reaven G, Hollenbeck C, Jeng C-Y, Wu MS, Chen YD (1988) Measurement of plasma glucose, free fatty acids, lactate, and insulin for $24 \mathrm{~h}$ in patients with NIDDM. Diabetes 37:1020-1024

25. Garvey WT, Olefsky JM, Rubenstein AH, Kolterman OG (1988) Day-long integrated serum insulin and C-peptide profiles in patients with NIDDM. Diabetes 37:590-599

26. Bolli GB, Gerich JE (1984) The 'dawn phenomenon'-a common occurrence in both insulin-dependent and non-insulin dependent diabetes mellitus. N Engl J Med 310:746-750

27. Yki-Jarvinen, Helve E, Sane T, Nurjhan N, Taskinen M-R (1989) Insulin inhibition of overnight glucose production and gluconeogenesis from lactate in NIDDM. Am J Physiol 256: E732-E739

28. Beebe CA, Van Cauter E, Shapiro ET et al (1990) Effect of temporal distribution of calories on the diurnal patterns of glucose levels and insulin secretion in NIDDM. Diabetes Care 13:748-755

29. Van Cauter E, Blackman JD, Roland D, Spire JP, Refetoff S, Polonsky KS (1991) Modulation of glucose regulation and insulin secretion by circadian rhythmicity and sleep. J Clin Invest 88:934-942

30. La Fleur SE, Kalsbeek A, Wortel J, Buijs RM (1999) A suprachiasmatic nucleus generated rhythm in basal glucose concentrations. J Neuroendcrinol 11:643-652

31. La Fleur SE, Kalsbeek A, Wortel J, Fekkes ML, Buijs RM (2001) A daily rhythm in glucose tolerance. A role for the suprachiasmatic nucleus. Diabetes 50:1237-1243

32. Pocal A, Lam TKT, Gutierrez-Juarez R et al (2005) Hypothalamic $\mathrm{K}_{\mathrm{ATP}}$ channels control hepatic glucose production. Nature 434:1026-1031

33. Kalsbeek A, La Fleur S, Van Heijningen C, Buijs RM (2004) Suprachiasmatic GABAergic inputs to the paraventricular nucleus control plasma glucose concentrations in the rat via sympathetic innervation of the liver. J Neurosci 24:7604-7613

34. Moore RY (1996) Neural control of the pineal gland. Behavioural Brain Res 73:125-130

35. Puchalski SS, Green JN, Rasmussen DD (2003) Melatonin effects on metabolism independent of gonad function. Endocrine 21:169-173

36. Ishikawa K, Shimazu T (1976) Daily rhythms of glycogen synthase and phosphorylase activities in rat liver: influence of food and light. Life Sci 19:1873-1878

37. Chu C, Sindelar DK, Neal DW, Allen EJ, Donahue EP, Cherrington AD (1998) Effect of a selective rise in sinusoidal norephrine on HGP is due to an increase in glycogenolysis. Am J Physiol 274:E162-E171

38. Dineen S, Alzaid A, Miles J, Rizza R (1995) Effects of normal nocturnal rise in cortisol on carbohydrate and fat metabolism in IDDM. Am J Physiol 268:E595-E603

39. Shamoon H, Hendler R, Sherwin RS (1980) Altered responsiveness to cortisol, epinephrine, and glucagon in insulininfused juvenile-onset diabetics; a mechanism for diabetic instability. Diabetes 29:284-291

40. Minorsky N (1962) Nonlinear oscillations. Van Nostrand, Princeton

41. Mackey MC, Glass L (1977) Oscillations and chaos in physiological control systems. Science 197:287-289

42. Glass L, Mackey MC (1979) Pathological oscillations resulting from instabilities in physiological control systems. Ann N Y Acad Sci 316:214-235 
43. Guyton AC, Crowell JW, Moore JW (1956) Basic oscillating mechanism of Cheyne-Stokes breathing. Am J Physiol 187:395-398

44. Lasota A, Mackey MC, Wazewska-Czyzewska M (1981) Minimizing therapeutically induced anemia. J Math Biol 13:149-158

45. Freiesleben D, Blasio B, Bak P, Pociot F, Karlsen AE, Nerup J (1999) Onset of type 1 diabetes. A dynamical instability. Diabetes 48:1677-1685

46. Ferrannini E, Nannipieri M, Williams K, Gonzales C, Haffner SM, Stern MP (2004) Mode of onset of type 2 diabetes from normal or impaired glucose tolerance. Diabetes 53:160-165
47. Radziuk J, Pye S (2004) Endogenous glucose production under basal and postprandial conditions in type 2 diabetes. Diabetologia 47(Suppl 1) A221 (Abstract)

48. Diabetes Prevention Program Research Group (2002) Reduction in the incidence of type 2 diabetes with lifestyle intervention or metformin. N Engl J Med 346:393-403

49. Basu R, Schwenk WF, Rizza RA (2004) Both fasting glucose production and disappearance are abnormal in people with "mild" and "severe" type 2 diabetes. Am J Physiol 287:E55-E62

50. Tappy L, Tounian P, Paquot N (1997) Autoregulation of endogenous glucose production in man. Biochem Soc Trans 25:11-13 